\title{
BMJ Open Prevalence and risk factors of adverse birth outcomes in the Pacific Island region: a scoping review protocol
}

\author{
Lydia Sandrah Kuman Kaforau (1) ,1,2 Gizachew Assefa Tessema,,3 \\ Jonine Jancey (D) , ${ }^{2}$ Gursimran Kaur Dhamrait (D) , ${ }^{4,5}$ Hugo Bugoro, ${ }^{1}$ G F Pereira ${ }^{2,6}$
}

To cite: Kaforau LSK, Tessema GA, Jancey J, et al. Prevalence and risk factors of adverse birth outcomes in the Pacific Island region: a scoping review protocol. BMJ Open 2021;11:e042423. doi:10.1136/ bmjopen-2020-042423

- Prepublication history for this paper is available online. To view these files, please visit the journal online (http://dx.doi. org/10.1136/bmjopen-2020042423).

Received 04 July 2020 Revised 16 March 2021 Accepted 22 March 2021

Deck for updates

(c) Author(s) (or their employer(s)) 2021. Re-use permitted under CC BY-NC. No commercial re-use. See rights and permissions. Published by BMJ.

For numbered affiliations see end of article.

\section{Correspondence to} Lydia Sandrah Kuman Kaforau; I.kaforau@postgrad.curtin. edu.au

\section{ABSTRACT}

Introduction Fetal growth restriction, preterm birth, low birth weight and stillbirth are adverse birth outcomes that are prevalent in low-income and middle-income settings such as the Pacific Island region. It is widely accepted that the excess burden of adverse birth outcomes is attributable to socioeconomic and environmental factors that predispose families to excess risk. Our review seeks to determine the prevalence of adverse birth outcomes in the Pacific Island region and to identify the risk factors of adverse birth outcomes in the Pacific Island region. Methods This scoping review will follow the five-staged Arksey and 0'Malley's framework and consultation with Solomon Islands' health stakeholders. A preliminary literature review was undertaken to understand the scope of the review. We will use Medical Subject Heading and keyword terms for adverse birth outcomes to search CINAHL, Medline, Scopus, ProQuest and Springer Link databases for articles published from 1 January 2000. The subsequent searches will be undertaken via Google Scholar and the internet browser to world health organisation and regional health organisations for published and unpublished reports on non-indexed studies. All articles retrieved will be managed with EndNote software. Eligible studies will be screened using Preferred Reporting Items for Systematic Reviews and Meta-Analyses flow chart for final selection. In the charting phase, we will extract the data into Excel spreadsheets. The results will be presented as numerical and thematic summaries that map risk factors and prevalence to the population and cultures of the Pacific Island region.

Ethics and dissemination Formal ethical approval is not required as primary or administrative data will not be collected. However, we will seek ethics approval for the stakeholder consultation from the Research Office of Curtin University and the Solomon Islands. The findings of this study will be published in peer-reviewed journals and presented in national and regional conferences and disseminated to stakeholders.

Ethics approval There will be no direct contact with human or patients in the case of the scoping review; therefore, no ethics will be required. However, we will seek ethical approval from the Research Ethics Office of Curtin University and the Health Research and Ethics Committee in the Solomon Islands for stakeholder consultation. Dissemination will be made through regional conferences and publication in peer-reviewed journals.

\section{Strengths and limitations of this study}

The review will provide information to help identify knowledge gaps and focal points for further investigation to progress towards evidenced-based maternal healthcare in the region.

- A strength of this study will be consultation with stakeholders (health professionals working in maternal and child health services) as they will provide insights into adverse birth outcomes at a community level.

- We may not be able to access studies published in languages other than English.

\section{INTRODUCTION}

Despite improvements in medical care and technology, the incidence of adverse birth outcomes remains a significant public health issue, particularly in low-income and middleincome countries (LMICs). ${ }^{12}$ Adverse birth outcomes include indicators for early gestation (preterm birth), fetal growth restriction and perinatal mortality. Preterm birth is the most well-accepted benchmark for morbidity attributable to early gestation and is defined as birth before 37 weeks of completed gestation. ${ }^{2}$ In LMICs, fetal growth restriction is indicated by its proxies ascertained at birth. ${ }^{3}$ These proxies include term low birth weight (LBW), defined as birth weight $<2500 \mathrm{~g}$ from 37 weeks of completed gestation, and small for gestational age (SGA), defined as weight in the lowest 10th centile for gestational age and sex or as a multiple of SD from the sexspecific population mean weight. LBW is also historically used as a proxy for preterm birth given the lack of information on gestational length. ${ }^{45}$ Fetal growth restriction is associated with infant mortality and morbidity. ${ }^{12}$ Stillbirth is the most commonly investigated mortality-related outcome and is defined as birth without signs of life from 28 weeks of completed gestation in LMIC. ${ }^{1}$ Both preterm birth and fetal growth restriction can 
significantly impact longer term physiological complications and well-being of children ${ }^{67}$ and are major risk factors for stillbirth.

The aetiologies of adverse birth outcomes are multifactorial and not entirely well understood. ${ }^{1}$ Evidence from studies conducted elsewhere show that socioeconomic, health, obstetric and biological factors are linked with adverse birth outcomes in high-income countries as well as LMICs. ${ }^{268-10}$ Moreover, evidence has also shown that environmental (non-genetic) risk factors are relatively more prevalent in LMICs resulting in higher infant mortality and morbidity in these countries. ${ }^{67}$ More than $96 \%$ of the 32 million LBW infants born globally each year occur in LMICs. ${ }^{8}$ Although adverse birth outcomes are reasonably well documented in some LMICs, such as India, ${ }^{11}$ studies in the Pacific Island region remain sparse.

The Pacific Island region broadly refers to a group of countries and territories that border the Pacific Ocean. ${ }^{12}$ The region, defined here as the LMICs and territories within the Melanesian, Polynesian and Micronesian subregions, are culturally and ethnically diverse, with varying degrees of economic development and living standards. ${ }^{12}$ The indigenous populations of the region are typically over-represented in national and global scales for disease burden for both communicable and non-communicable diseases. ${ }^{12}$ Health indicators also vary considerably across this region; for example, the infant mortality rate in Papua New Guinea is 50 per 1000 births compared with 20 per 1000 births in Fiji. ${ }^{13}$ Similarly, LBW and SGA also vary within and between countries of the region with reported prevalence inconsistent and underreported. ${ }^{14}$ A review in 2013 estimated a period prevalence of $8 \%$ for preterm birth, $10 \%$ for LBW and $19 \%$ for SGA in the broader region of Oceania, ${ }^{15}$ but these prevalence are not well established for the Pacific Island region specifically. Moreover, although it is estimated that $98 \%$ of stillbirths occur in LMICs, ${ }^{16}$ there are no high-quality estimates for stillbirth prevalence in the Pacific Island region. In the last two decades, there has been a substantial decline in infant and child mortality by approximately $50 \%$ in more than half of the Pacific Island countries and territories. ${ }^{14}$ However, the extent of such improvements remains uncertain due to poor data quality and coverage and impacting cultural factors.

Deficiency in the provision of basic health services such as antenatal care and delivery services, infrastructure, telecommunication and transportation are pertinent contributors to the burden of adverse birth outcomes in the Pacific Island region. ${ }^{17}$ Notably, more than $60 \%$ of the population in the region live in rural areas. ${ }^{18}$ Factors such as access to healthcare, diet and substance use vary considerably. There is some indication that levels of alcohol consumption and tobacco and substance use (including betel or areca nut) may be among the highest globally. ${ }^{19-23}$

The aim of this scoping review is to synthesise available results from studies on the prevalence and risk factors of adverse birth outcomes in the Pacific Island region.
Knowledge of the burden of adverse birth outcomes and key risk factors will provide policy makers and healthcare practitioners working in the region with evidence that can be used to inform strategies to achieve reductions in adverse birth outcomes and improve overall perinatal health. These research findings will help to design targeted interventions and better allocate resources to where they are needed. Additionally, findings of the review will inform future aetiological research on the effect of risk factors of adverse birth outcomes in the region.

\section{METHODS}

This scoping review will follow the Joanna Briggs Institute Reviewers Manual $^{24}$ derived from Arksey and O'Malley's five-staged methodological Framework ${ }^{25}$ and further developed by Levac et al..$^{26}$ Briefly, this includes explicit specification of research questions, reproducible methods to identify relevant studies, transparent declarations of inclusion and exclusion criteria, documented collation of data and standardised summarisation and reporting of results. The scoping review will not involve patients and the public as data will be sourced from primary studies. However, we will also include an optional stage six of stakeholder consultation for additional insights. The stakeholder consultation exercise will only be involving doctors, midwives and nurses who work directly with pregnant women. Ethics and consent will be sought from respective authorities and clinicians. Our reporting will also be compliant with Preferred Reporting Items for Systematic Reviews and Meta-Analyses extension for scoping reviews checklist. ${ }^{24}$ A preliminary literature review was undertaken to understand the extent of literature on exposures of risk factors of adverse birth outcomes in the Pacific Island region to determine an appropriate search timeframe. Thus, the scoping review will be conducted between December 2020 and February 2021.

\section{Stage 1: specification of the research question}

We will first identify the research question. A preliminary literature review was undertaken to understand the extent of literature on exposures of risk factors of adverse birth outcomes in the Pacific Island region to determine an appropriate search timeframe. This stage will allow the formulation of the research questions for the study. The broad research questions are: what is the prevalence of the adverse birth outcomes in the Pacific Island region?, and what are the risk factors of adverse birth outcomes in the Pacific Island region? The indigenous population of the region are broadly classified as Melanesian, Polynesian and Micronesians, each with their own diverse historical roots and cultures. ${ }^{12}$ Such diversity is accompanied by differences in economic development and living standards, causing a wide variation in health outcomes between populations. ${ }^{12}$ Consequently, this review will also describe the prevalence and risk factors by subpopulation group. 
Table 1 Inclusion and exclusion criteria

\begin{tabular}{ll} 
Inclusion criteria & Exclusion criteria \\
\hline All studies and articles irrespective of their designs. & Studies on Pacific Islanders living in countries outside the region. \\
Primary and secondary studies. & Studies on Non-Pacific Islanders living in the Pacific Islands. \\
Population and inferential-based studies. & \\
- Mother and infants' populations. & \\
- 21 Pacific Island countries and territories. &
\end{tabular}

\section{Stage 2: identifying relevant studies}

The second stage of the review aims to identify the relevant studies through the eligibility criteria and search strategies involved. The Arksey and O'Malley's methodological framework ${ }^{25}$ uses population-concept-context. For this review, the population is defined as all women of childbearing age (15-49 years old) who gave birth in the Pacific Island region and infants from these births; concept is the prevalence and risk factors for adverse birth outcomes (low birth weight, preterm birth, small for gestational age or fetal growth restriction, stillbirths and miscarriage); and context is defined geographically as all 21 countries and territories in the region.

\section{Inclusion and exclusion criteria}

We will include all studies and articles irrespective of their study design. We will incorporate all studies that report risk factors and their associations with one or more of the adverse birth outcomes in the Pacific Island region arising during pregnancy but observed at the separation of the fetus from the mother or shortly afterwards. We will include studies that will provide estimates of the prevalence rates and risk factors of adverse birth outcomes. That include inferential studies that aimed to estimate the prevalence and identify associated risk factors such as intervention and observational studies. Our review will also include descriptive population-based studies such as the Demographic Health Surveys and other surveys. We will include studies from the 21 sovereign island states and territories of the region, namely: American Samoa, Cook Islands, Easter Islands, Federated States of Micronesia, Fiji, Guam, Kiribati, Mariana Islands, Marshall Islands, Nauru, New Caledonia, Niue, Palau, Papua New Guinea, Samoa, Solomon Islands, Tahiti, Tokelau, Tonga, Tuvalu, Vanuatu, and Wallis and Futuna. ${ }^{14}$ Both primary and secondary analytical studies published in peer-reviewed journals and grey literature as government reports will be included. Studies published in English from the year 2000 to February 2021 will be included. Table 1 illustrates a summary of the inclusion and exclusion criteria for the study.

\section{Search strategy}

The search strategy will follow the three-stage search process outlined by the Joanna Briggs Institute. ${ }^{27}$ The first stage will include an initial search using key concept terms that will be undertaken in CINAHL and Medline to identify Medical Subject Heading (MeSH) or text terms contained within the titles and abstracts of articles. The key concept terms are adverse birth outcomes, pregnancy risk factors and Pacific Island region. Table 2 outlines the grid of key concepts and terms.

In the second stage of the search, all MeSH terms, key concept terms and their synonyms will be combined with Boolean operators, truncations and wildcards to generate search strings and will be applied across the selected databases. The following electronic databases will be searched: CINAHL, Medline, ProQuest, SpringerLink and Scopus. As all databases have different search protocols, we will ensure to follow each of their guidelines accordingly. In the second stage, we will carry out two levels of searches. The first level will use general key concept terms and their synonyms combined with MeSH terms identified. An example of general search string designed for CINAHL is as follows: ("adverse birth outcome*" OR "poor birth outcome*" OR "preterm birth*" OR "premature birth*" OR "Poor fetal growth*" OR "fetal growth restriction*" OR "intrauterine growth retardation" OR "growth retardation" OR "small baby*" OR "very small baby*"OR "low birth weight" OR "low birthweight" OR "very low birth weight" OR "very low birthweight" OR "extremely low birth weight" OR "extremely low birthweight" OR "stillbirth" OR "still birth" OR MH "pregnancy outcome*" OR MH "infant very low birth weight" OR MH "outcome* of pregnancy outcome*" OR "poor pregnancy outcome*" OR MH "risk factor*” OR MH "pregnancy risk*” OR MH "high risk*” OR MH "pregnancy in adolescence*” OR MH "pregnancy risk*) AND ("Pacific Island*” OR “Oceania" OR "South Pacific Island*" OR "Pacific Island country*" OR "MH Pacific Island*”). prematurity") AND ("pregnancy risk factor*" OR "adverse

Table 2 Grid of key concepts and terms

\begin{tabular}{|c|c|c|c|c|}
\hline Concept 1 & & Concept 2 & & Concept 3 \\
\hline Adverse birth outcomes & AND & Pregnancy risk factors & AND & Pacific Island region \\
\hline
\end{tabular}


Table 3 CINAHL searches

Key concepts and terms

The following key concepts were identified from the topic.

\section{Concept 1}

Adverse birth outcomes OR

\section{Concept 2}

Pregnancy risk factors

\section{Concept 3}

Pacific Island region

$\mathrm{MeSH}$ and subject headings identified.

\section{Key concept terms}

Adverse birth outcomes

\section{CINAHL}

MH "Pregnancy outcome*" OR MH "Infant very Low birth weight" OR MH "Outcome* of prematurity"

Pregnancy risk factors

MH "Risk factor" OR MH "Pregnancy risk" OR MH "High risk"” OR MH "Pregnancy in adolescence" OR MH "Pregnancy risk"”

Pacific Island region $\mathrm{MH}$ "Pacific Island"”

Search strings developed

\section{Key concept and general terms and synonyms search string}

("adverse birth outcome*" OR "poor birth outcome*" OR "preterm birth"” OR "premature birth*" OR "Poor fetal growth"” OR "fetal growth restriction"” OR "intrauterine growth retardation" OR "growth retardation" OR "low birth weight" OR "low birthweight" OR "very low birth weight" OR "very low birthweight" OR "extremely low birth weight" OR "extremely low birthweight" OR "stillbirth" OR "still birth") AND ("pregnancy risk factor*" OR "adverse pregnancy outcome "* OR "poor pregnancy outcome*") AND ("Pacific Island"” OR "Oceania" OR "South Pacific Island"” OR "Pacific Island country")

\section{MeSH terms search string}

(MH "pregnancy outcome*" OR MH "infant very low birth weight" OR MH "outcome* of prematurity") AND (MH "risk factor" OR MH "pregnancy risk" OR MH "high risk" OR MH "pregnancy in adolescence*” OR MH "pregnancy risk") AND ("MH Pacific Island")

\section{General and MeSH terms combined search string}

("adverse birth outcome*" OR "poor birth outcome*" OR "preterm birth"” OR "premature birth" OR "Poor fetal growth" OR "fetal growth restriction*" OR "intrauterine growth retardation" OR "growth retardation" OR "small baby" OR "very small baby*"OR "low birth weight" OR "low birthweight" OR "very low birth weight" OR "very low birthweight" OR "extremely low birth weight" OR "extremely low birthweight" OR "stillbirth" OR "still birth" OR MH "pregnancy outcome*" OR MH "infant very low birth weight" OR MH "outcome* of prematurity") AND ("pregnancy risk factor"” OR "adverse pregnancy outcome *" OR "poor pregnancy outcome*" OR MH "risk factor" OR MH "pregnancy risk" OR MH "high risk" OR MH "pregnancy in adolescence"” OR MH "pregnancy risk") AND ("Pacific Island"” OR "Oceania" OR "South Pacific Island"” OR "Pacific Island country" OR "MH Pacific Island*")

\section{Specific and MeSH terms combined search string}

("preterm birth*" OR "premature birth" OR "Poor fetal growth*" OR "fetal growth restriction*" OR "intrauterine growth retardation" OR "growth retardation" OR "small baby*" OR "very small baby*" OR "low birth weight" OR "low birthweight" OR "very low birth weight" OR "very low birthweight" OR "extremely low birth weight" OR "extremely low birthweight" OR "stillbirth" OR "still birth" OR MH "pregnancy outcome" OR MH "infant very low birth weight" OR MH "outcome* of prematurity") AND ("malaria in pregnancy" OR "anaemia in pregnancy" OR "substance use" OR "alcohol use" OR "betel nut use" OR "areca nut use" OR "tobacco use" OR "cigarette use" OR "maternal obesity" OR "maternal malnutrition" OR "maternal undernutrition" OR "teenage pregnancy") AND ("American Samoa" OR "Cook Island"” OR "Easter Island*" OR "Federated States of Micronesia" OR "Fiji" OR "Guam" OR "Kiribati" OR "Mariana Island"” OR "Marshall Island"” OR "Nauru" OR "New Caledonia" OR "Niue" OR "Palau" OR "Papua New Guinea" OR "Samoa" OR "Solomon Island”" OR "Tahiti" OR "Tokelau" OR "Tonga" OR "Tuvalu" OR "Vanuatu" OR "Wallis and Futuna")

Filter/limiter used

Year inclusion 2000 current

Full-text articles

English language $\mathrm{MeSH}$

MeSH, Medical Subject Headings.

Similarly, a specific search with more precise key terms or specific risk factors will narrow the search down for each country. Specific search terms will be identified through the initial literature review to understand the specific risk factors within the population. An example of specific key and $\mathrm{MeSH}$ terms and search strings also designed for 
CINAHL is as follows: ("preterm birth*" OR "premature birth*" OR "Poor fetal growth*" OR "fetal growth restriction*" OR "intrauterine growth retardation" OR "growth retardation" OR "small baby*" OR "very small baby*" OR "low birth weight" OR "low birthweight" OR "very low birth weight" OR "very low birthweight" OR "extremely low birth weight" OR "extremely low birthweight" OR "stillbirth" OR "still birth"OR MH "pregnancy outcome*" OR MH "infant very low birth weight" OR MH "outcome* of prematurity") AND ("malaria in pregnancy" OR "anaemia in pregnancy" OR "substance use" OR "alcohol use" OR "betel nut use" OR "areca nut use" OR "tobacco use" OR "cigarette use" OR "maternal obesity" OR "maternal malnutrition" OR "maternal undernutrition" OR "teenage pregnancy") AND ("American Samoa" OR "Cook Island*" OR "Easter Island*" OR "Federated States of Micronesia" OR "Fiji" OR "Guam" OR "Kiribati" OR "Mariana Island*" OR "Marshall Island*" OR "Nauru" OR "New Caledonia" OR "Niue" OR "Palau" OR "Papua New Guinea" OR "Samoa" OR "Solomon Island*" OR "Tahiti" OR "Tokelau" OR "Tonga" OR "Tuvalu" OR "Vanuatu" OR "Wallis and Futuna"). Table 3 illustrates a comprehensive search of general and specific search terms combined with $\mathrm{MeSH}$ that will be applied to CINAHL database.

In the third stage of the search, we will assess the reference lists of studies initially retrieved in order to identify any relevant studies that have not been identified by the electronic database searches. Additional searches will also be conducted to identify non-indexed studies and manually searching thesis repositories, Google Scholar and Google for regional health organisation websites. The online sources that we will search include the UNICEF, WHO, Pacific community and individual countries health websites.

\section{Stage 3: study selection}

At this stage, we will screen and select the studies. During the primary review, we will consolidate all studies retrieved, remove all duplicates and remove studies that do not correspond to the Population Concept Context criteria. ${ }^{28}$ Next, we will screen the titles and abstracts of articles after importing all records retrieved from databases and webbased searches into EndNote. Two reviewers (LK and GT) will be conducting the study selection and data abstraction. ${ }^{26}$ Any uncertainty with the title and abstract will go through full-text review. Any uncertainty reached on any article will be discussed with the broader research team. If consensus is not reached, articles will be excluded from the review. All remaining articles will go through full-text screening, following the Preferred Reporting Items for Systematic Reviews and Meta-Analyses flow chart, ${ }^{29}$ and final articles will proceed to the final review.

\section{Stage 4: charting data}

Data charting will involve data extraction and documenting from the final articles selected. During the data extraction, all results will be entered into Excel spreadsheets alongside standard bibliographic information that includes author(s), year

\section{Box 1 Data extraction table}

Main category
a. Author(s).
b. Year of publication.
c. Origin/country study was conducted.
d. Study design.
e. Aims/purpose.
f. Sampling strategy.
g. Study population.
h. Sample size.
i. Methodology.
j. Intervention/exposure type (if applicable) and comparison group (if
k. Duration of the exposure/intervention (if applicable).
I. Outcomes assessment and method to assess associations (if
m. Key findings that relate to the scoping review question/s.

of publication, origin or country of origin, aims and purpose, study population, methodology, intervention type, intervention duration, outcomes and details and key findings. Box 1 outlines the standard bibliographic information. ${ }^{27}$ For each article, reviewed key information to be retrieved will be risk factors matched to birth outcomes and prevalence to the specific context of the region. The framework will be pilot tested by the reviewers to ensure that it is consistent with data charting and the study aims and objectives. Charting of data will be an iterative process of screening and extracting data that will be done mostly by the principal investigator. Any arising questions and uncertainty during the process will be discussed with the research team to reach an agreement.

\section{Stage 5: collating, summarising and presenting the results} In stage five, tabular presentation of the findings will be mapped from data extracted from the selected articles, as outlined (see Box 1) and guided by Arskey and O'Malley. ${ }^{25}$ Findings will be presented quantitatively in aggregated forms figure and qualitatively as thematic narrative summaries, all of which will reflect the study objectives. ${ }^{25}$ The results of the studies will not be compared but presented as a body of evidence. We expect to map a wide range of risk factors, prevalence and the different adverse birth outcomes against the countries' ethnic and geographical diversity to provide the first such body of literature for the region.

\section{Stage 6: stakeholder consultation}

A consultation exercise will be conducted online with relevant health professionals in the Solomon Islands, including midwives, paediatric nurses, obstetricians and paediatricians identified through contacts and purposive and snowball sampling. This stage aims to validate findings from this study and to add additional insights and recommendations from their perspectives. Consultation will be undertaken at the completion of the article review. The exercise will involve the collection of quantitative and qualitative feedback from clinicians who work with pregnant mothers and infants to obtain their knowledge and experience of risk factors and birth 
outcomes in the Solomon Islands from a clinical perspective. Ten health professionals working with pregnant woman and infants will be consulted. Selection will be made by purposeful and snowball sampling.

\section{Stage 7: patient and public involvement}

No patient involved.

\section{Author affiliations}

${ }^{1}$ Faculty of Nursing, Medicine and Health Sciences, Solomon Islands National University, Honiara, Solomon Islands

${ }^{2}$ School of Public Health, Curtin University Faculty of Health Sciences, Perth, Western Australia, Australia

${ }^{3}$ School of Public Health, University of Adelaide, The University of Adelaide Faculty of Health Sciences, Adelaide, South Australia, Australia

${ }^{4}$ Department of Public Health, Telethon Kids Institute, Nedlands, Western Australia, Australia

${ }^{5}$ School of Population and Global Health, The University of Western Australia Faculty of Health and Medical Sciences, Perth, Western Australia, Australia

${ }^{6}$ Norwegian Institute of Public Health, Centre for Fertility and Health (CeFH), Norwegian Institute of Public Health, Oslo, Norway

Twitter Lydia Sandrah Kuman Kaforau @MorningDew Kaforau and Jonine Jancey @joninejancey

Acknowledgements The authors would like to acknowledge the invaluable support and input from the librarian (Faculty of Health Science of Curtin University), the reviewers (BMJ Open) and our team of peer reviewers.

Contributors LSKK, GFP, GAT and JJ: study inception, conceptualisation and design, LSKK: drafted the first version and conducted the preliminary searches, collating all inputs reiteratively and revision of the manuscript; GAT, JJ, HB, GKD and GFP: critically reviewed the manuscript. All authors read and approved the final version.

Funding Article Processing Charge waiver claim 00164914. Full waiver grant number 00D0YaQIK._5001v1P90VA. National Health and Medical Research Council Grants. \#1099655 to GFP, \#1173991 to GFP and \#1195716 to GAT. Research Council of Norway Grants \#262700 to GP.

Competing interests None declared.

Patient and public involvement Patients and/or the public were involved in the design, or conduct, or reporting, or dissemination plans of this research. Refer to the Methods section for further details.

Patient consent for publication Not required.

Provenance and peer review Not commissioned; externally peer reviewed.

Open access This is an open access article distributed in accordance with the Creative Commons Attribution Non Commercial (CC BY-NC 4.0) license, which permits others to distribute, remix, adapt, build upon this work non-commercially, and license their derivative works on different terms, provided the original work is properly cited, appropriate credit is given, any changes made indicated, and the use is non-commercial. See: http://creativecommons.org/licenses/by-nc/4.0/.

\section{ORCID iDs}

Lydia Sandrah Kuman Kaforau http://orcid.org/0000-0002-7894-2896

Jonine Jancey http://orcid.org/0000-0002-7894-2896

Gursimran Kaur Dhamrait http://orcid.org/0000-0002-5191-211X

\section{REFERENCES}

1 Weng Y-H, Yang C-Y, Chiu Y-W. Risk assessment of adverse birth outcomes in relation to maternal age. PLoS One 2014;9:e114843.

2 Berhan T, Andargachew K. Prevalence of adverse birth outcome and associated factors among women who delivered in Hawassa town governmental health institutions, South Ethiopia, in 2017. Reproductive Health 2018;15:1-10.

3 Sayers SM, Lancaster PAL, Whitehead CL. Fetal Growth Restriction: Causes and Outcomes. In: Quah SR, ed. International encyclopedia of public health. Second Edition. Oxford: Academic Press, 2017: 132-42.
4 Wachamo TM, Bililign Yimer N, Bizuneh AD, Tesfahun MW, Nigus BY, Asmamaw DB. Risk factors for low birth weight in hospitals of North Wello zone, Ethiopia: a case-control study. PLoS One 2019;14:e0213054.

5 Tampah-Naah A, Anzagra L, Yendaw E. Factors correlate with low birth weight in Ghana. Br J Med Med Res 2016;16:1-8.

6 Su D, Samson K, Garg A, et al. Birth history as a predictor of adverse birth outcomes: evidence from state vital statistics data. Prev Med Rep 2018;11:63-8.

7 Adane AA, Ayele TA, Ararsa LG, et al. Adverse birth outcomes among deliveries at Gondar university Hospital, Northwest Ethiopia. BMC Pregnancy Childbirth 2014;14:90.

8 Tsegaye B, Kassa A. Prevalence of adverse birth outcome and associated factors among women who delivered in Hawassa town governmental health institutions, South Ethiopia, in 2017. Reprod Health 2018;15:193-93.

9 Chawanpaiboon S, Vogel JP, Moller A-B, et al. Global, regional, and national estimates of levels of preterm birth in 2014: a systematic review and modelling analysis. Lancet Glob Health 2019;7:e37-46.

10 Charlton KE, Russell J, Gorman E, et al. Fish, food security and health in Pacific island countries and territories: a systematic literature review. BMC Public Health 2016;16:285.

11 Dongarwar D, Salihu HM. Place of residence and inequities in adverse pregnancy and birth outcomes in India. Int J MCH AIDS 2020;9:53-63.

12 Horwood PF, Tarantola A, Goarant C, et al. Health challenges of the Pacific region: insights from history, geography, social determinants, genetics, and the microbiome. Front Immunol 2019;10:2184

13 OECD, Organisation WH. Health at a glance: Asia/Pacific 2018: measuring progress towards universal health coverage. ParisOECD Publishing; 2018: 1-134. https://doi.org/10.1787/health_glance_ap2018-en [Accessed 29.03.21].

14 Linhart C, Carter K, Sorchik R. Trends in neonatal and infant mortality for Pacific island states. Secretariat of the Pacific Community Cataloguing-in-publication data Secretariat of the Pacific Community; 2015: 1-90. https://sdd.spc.int/en/reports-manuals/101pacific-infant-and-neonatal-mortality-trends-report

15 Lee ACC, Katz J, Blencowe $\mathrm{H}$, et al. National and regional estimates of term and preterm babies born small for gestational age in 138 lowincome and middle-income countries in 2010. Lancet Glob Health 2013;1:e26-36.

16 Yakoob MY, Lawn JE, Darmstadt GL, et al. Stillbirths: epidemiology, evidence, and priorities for action. Semin Perinatol 2010;34:387-94.

$17 \mathrm{WHO}$. Country cooperation stratergy at a glance, Pacific island countries WHO country page; 2013: 1-2. http://www.who.int/ countryfocus [Accessed 29 Mar 2021].

18 Andrew NL, Bright P, de la Rua L, et al. Coastal proximity of populations in 22 Pacific island countries and territories. PLoS One 2019;14:e0223249.

19 Quinn B, Peach E, Wright CJC, et al. Alcohol and other substance use among a sample of young people in the Solomon Islands. Aust $N$ Z J Public Health 2017;41:358-64.

20 Pratt $S$. The challenge of betel nut consumption to economic development: a case of Honiara. Solomon Islands 2014;21:103.

21 Jarawan E, Carpio C. Health challenges in the small island developing countries of the Pacific and the Caribbean. OnlineWorld Bank; unknown: 1-37. https://www.worldbank.org/content/dam/ Worldbank/Health

22 De Silva M, Panisi L, Brownfoot FC, et al. Systematic review of areca (betel nut) use and adverse pregnancy outcomes. Int J Gynaecol Obstet 2019;147:292-300.

23 Berger KE, Masterson J, Mascardo J, et al. The effects of chewing betel nut with tobacco and Pre-pregnancy obesity on adverse birth outcomes among Palauan women. Matern Child Health $J$ 2016;20:1696-703.

24 Aromataris E, Munn Z. JBI reviewer's manual. 488. Adelaide, Australia: Joana Briggs Institute, 2020.

25 Arksey H, O'Malley L. Scoping studies: towards a methodological framework. Int J Soc Res Methodol 2005;8:19-32.

26 Levac D, Colquhoun H, O'Brien KK. Scoping studies: advancing the methodology. Implement Sci 2010;5:69.

27 Peters M, Godfrey C, Khalil H. 2017 guidance for the conduct of JBI scoping reviews. Joana Briggs Inst Rev Man 2017;13:141-6.

28 Halas G, Schultz ASH, Rothney J, et al. A scoping review protocol to map the research foci trends in tobacco control over the last decade. BMJ Open 2015;5:e006643-e43.

29 Moher D, Liberati A, Tetzlaff J, et al. Preferred reporting items for systematic reviews and meta-analyses: the PRISMA statement. PLoS Med 2009;6:e1000097. 